\title{
Broadening IR applications through using spectral filters
}

\author{
by G. Pas
}

Inframetrics-Europe, Mechelsesteenweg 277, B-1800 Vilvoorde, Belgium

Tel. (32) 225790 30, Fax. (32) 225790 39, e-mail: guy.pas@inframetrics.be

\begin{abstract}
Some materials we look at with an infrared camera can appear "transparent", so we actually see through them rather than looking at them. In such cases, you end up measuring the temperature of objects behind the target you intended to measure, rather than the target itself. Examples of these types of materials include glass, plastics and many gasses. Other applications involve situations where we need to see through a semi-transparent material or gas to get at the object of interest. Examples of this type of situation include looking through furnace gasses or through a glass or plastic package to see what's going on inside. Knowing the spectral characteristics of the material and selecting the proper filter-IR camera combination can solve many difficult measurement situations.

The infrared camera must have the capability of utilizing specific infrared filters to tune the IR system for viewing and measuring objects involving IR transparent materials. Understanding which filter to use and its limitations is critical to getting good results and making proper decisions from the data.
\end{abstract}

\section{Understanding an IR Camera's Spectral Capabilities}

Many people in the IR community purchase and use IR cameras with no knowledge or attention paid to the spectral sensitivity of their cameras. In many applications, the spectral characteristics are not important and have little effect on the results obtained by the camera. However, some applications depend greatly on the wavelength that is being viewed, to the point that the object may appear completely transparent or opaque, which may well provide extremely erroneous results. Exploiting the spectral characteristics of your camera together with the IR absorption/transmission characteristics of the target you are viewing, is the way to get the results you want.

The first step in this process is obtaining the spectral characteristics of your IR camera's optical system, a combination of the IR detector, windows and lenses. Typically, the IR detector has far broader spectral sensitivity than the other components. The lenses and windows are coated with IR antireflective coatings, limiting the wavelength region impinging on the detector. This is done to maximize camera performance for temperature measurement situations.

Nearly all IR systems in use today fall into one of two categories, long wavelength and short wavelength. A "shortwave" camera is one that sees in the "midwave" IR band, typically in the spectral region from $2 \mu \mathrm{m}$ to $5 \mu \mathrm{m}$. This is an area where you need to be a little careful.

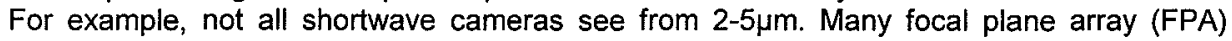
based IR cameras see over a narrower region, typically from $3.6 \mu \mathrm{m}$ to $5.0 \mu \mathrm{m}$. This is a result of the specific FPA detector material's responsivity and a desire by some manufacturers to reduce the effects of the sun at the shorter wavelengths during outdoor surveys. Knowing the exact limitations of the detector/lens system you are using is important, since it defines ultimately what IR filters you can apply to that system. You cannot, for example, apply an IR filter that operates outside the IR spectral responsivity of your IR camera.

\section{Where to use IR filters?}

The first challenge in selecting an IR filter can be determining whether or not a filter can help the measurement situation. One rule of thumb is that filters typically do not improve the emissivity of an object but rather change the transmissivity of an object or environment. There are a few exceptions to this rule, as with glass, which we will discuss later. However, 
in most cases, you have an object, which you are seeing through, which you want to measure, as is the case with most plastic films. Or, you have a situation where you want to see through something to measure an object inside an environment, as is the case when measuring tube temperatures through gaseous flames in a furnace. In either case, the first step is to recognize that transmissivity is an issue with regard to accurate temperature measurement. Then you need to proceed to analyzing the transmissive spectral characteristics of the object you are dealing with.

\section{Characterizing IR spectral transmissivity}

Characterizing the IR spectral transmissivity of an object is important because it provides the key details necessary to overcoming the barriers to measuring the object's temperature with IR. Spectral transmissivity is typically measured by a spectral scan of the object. A spectral scan is a graph depicting relative transmission versus wavelength for an object in a controlled environment. When the goal is to measure objects, which are normally transmissive, like plastic films, you need to look to the areas of the spectral scan, which indicate low IR transmission (or conversely, high IR absorption). Looking at the object in these specific wavelengths will make the

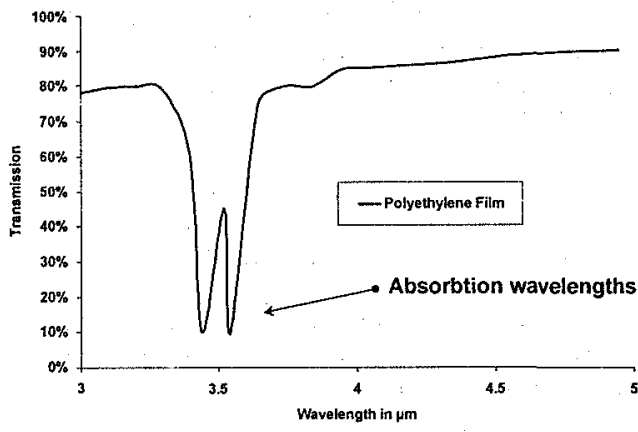

Fig. 1. Spectal transmission of Polyethylene plastic film Valleys indicate wavelengths where will appear opaque object appear "opaque", thus eliminating the effects of "seeing through" the object.

When trying to look through a semi-transparent object or environment, like furnace gasses, you need to look for areas of high IR transmission in the spectral scan. Looking in these specific wavelengths will make the object or environment look "transparent", allowing the camera to see through the environment without effect from the temperature of what you are looking through.

Determining the actual IR transmission characteristics of an object can be done in one of two ways. If you are dealing with a fairly common material, (glass, plastic, furnace flames) you can rely on previously established data on these items and simply call on existing spectral data. When dealing with unique materials or materials of varying thickness, you need to collect actual data from a sample of the object by performing a spectral scan using an IR spectrometer.

\section{Matching object characteristics to camera/filter solutions}

The key to success with IR filters is to match the object's spectral characteristics to an appropriate filter, which has been calibrated inside the IR camera. Selecting the right filter involves the following steps:

1. Determine what effect the IR filter needs to have on the object to solve the measurement problem, i.e. turn a semi transparent object into an opaque object or a fully transparent object.

2. Locate the appropriate regions on the spectral scan of the object for the desired effect, i.e. locate regions of low transmission for objects you want to look opaque and locating regions of high transmission for objects you want to appear transparent.

3. Isolate IR transmissive or absorptive areas that are within the IR spectral range of your IR camera. For example, some plastics have absorption bands at $3.4 \mu \mathrm{m}$ and at $6.9 \mu \mathrm{m}$. You would need to work with the $3.4 \mu \mathrm{m}$ absorption band if you had a shortwave IR camera, since $6.9 \mu \mathrm{m}$ is not within the normal shortwave band. 
4. Select an IR filter, which matches the IR transmission or absorption band you have selected, i.e. for plastics, a filter operating at $3.4 \mu \mathrm{m}$ is ideal.

5. Have the IR filter calibrated with your IR camera by the camera manufacturer.

6. Characterize the performance of the camera with the filter (accuracy and sensitivity will be affected due to a reduction in energy going to the detector)

\section{Measuring thin film plastics}

A common application that is well suited to IR filters is that of measuring thin film plastics. Since the process of making thin plastic film itself is highly temperature critical, it is imperative to evaluate both the temperature and the uniformity of the plastic as it exits the extruder or web process. The product is typically moving at high speeds which preclude the use of contact temperature methods.

Most plastic films have spectral characteristics similar to polyethylene (depicted in the figure below) which is transmissive in both the shortwave and longwave IR regions. Measuring thin film plastics can be challenging since without using a filter, you see "through" the plastic and measure the objects behind the plastic, rather than the plastic itself.

By analyzing the IR spectral characteristics of most plastics, you will find that a significant absorption band exists at $3.4 \mu \mathrm{m}$. This absorption band is a region where the plastic will appear opaque to the IR camera, allowing the camera to measure the temperature of the plastic and not the background. The width of this absorption

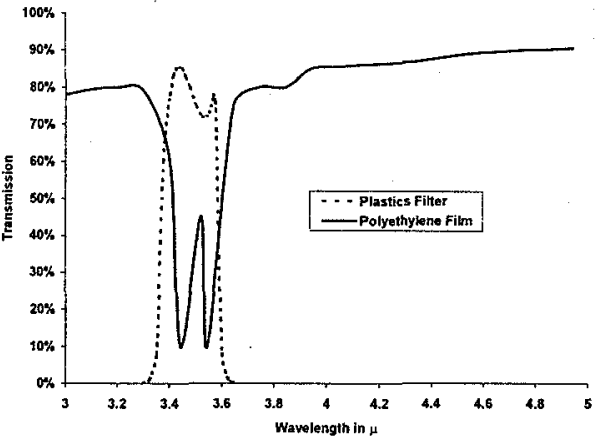

Fig. 2. Spectral transmission of Polyethylene plastic film with matching spectral response of ThermaCAM ${ }^{\mathrm{TM}}$ plastics filter band will vary with the thickness of the plastic being measured. The thicker the plastic, the deeper the absorption band will be. Utilizing a filter, which allows only the energy from a region around $3.4 \mu \mathrm{m}$, is ideal for this type of plastic film.

It is important to note that if you are using an FPA camera for this type of application, you must make sure that the basic detector has the capability of imaging a spectral range, which includes the plastic absorption band. Many Platinum Silicide based FPA cameras have a factory installed "cut-on" filter which removes all energy below 3.6 $\mu \mathrm{m}$. This type of camera cannot be used in plastics applications since a $3.4 \mu \mathrm{m}$ filter would be outside of the camera's spectral imaging capabilities. 


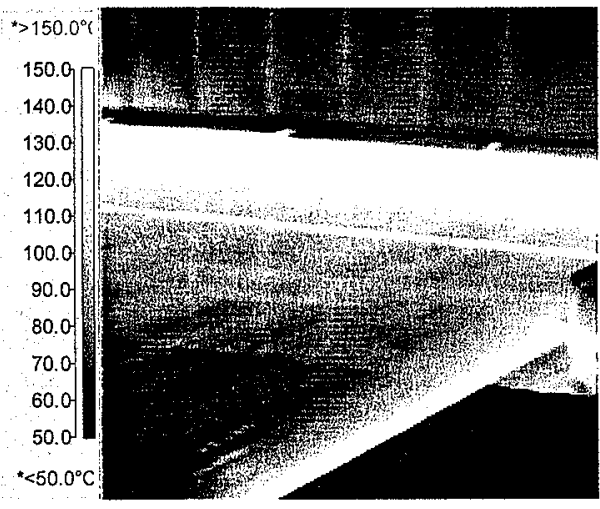

Fig. 3. View of Polyethylene plastic film process without plastics filter illustrates transparent nature of the material

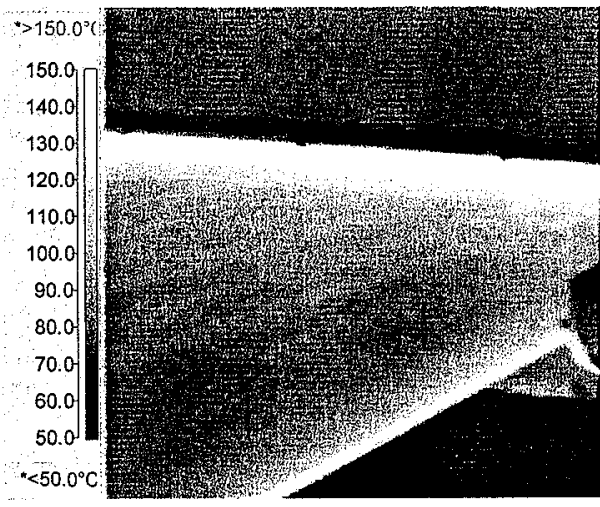

Fig. 4. View of Polyethylene plastic film process with plastics filter illustrates how filter eliminates transparent nature of the material

\section{Measuring through furnace flames}

Having the ability to accurately determine the temperature of process tubes in a noncontact manner provides the capability to find cool spots due to "coking", monitor tube temperatures during steam de-coking operations and for performing thermocouple verifications. A critical aspect of having accurate readings in this application is to remove the effects of the hot gaseous environment the camera has to measure through. Spectral studies of a variety of furnace gasses have revealed that a transmission region exists at $3.9 \mu \mathrm{m}$ in most cases.

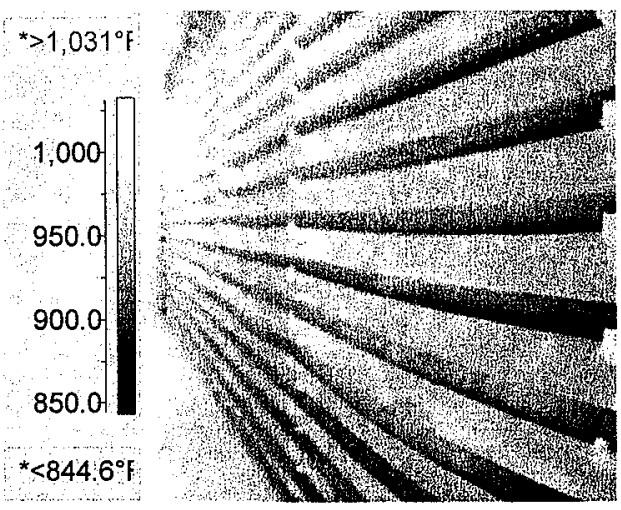

Fig. 5. Image of furnace tubes with flame filter illustrates regions with coking while providing accurate temperature measurement

Measuring temperatures in a furnace view port presents several challenging stresses on a thermal imaging system. These stresses typically revolve around the effects of the high radiant and convective thermal loads that are put on the camera optics and electronics. It is not uncommon for an IR camera body and lens to heat up to temperatures in excess of $60^{\circ} \mathrm{C}$ in a matter of a couple of minutes when looking inside a typical furnace port. For these reasons, it is essential that the IR camera be designed for these types of applications.

Most IR FPA cameras available today have high temperature measurement capabilities available through flame filtration, where an emphasis has been applied to accurate temperature measurement of calibrated sources in a laboratory environment. However, as with the furnace tube application, the environment is everything but a laboratory and the target is much larger than a typical calibrated temperature source. When equipped with a $3.9 \mu \mathrm{m}$ flame suppression filter, an IR camera's provides the most accurate measurements for this application. 


\section{Measurements involving glass}

Many products or processes involve working with hot glass. Measuring the temperature of the glass surface and/or the objects encased inside the glass can be critical, but very difficult to accomplish. The most common example of this is in the light bulb industry.

Measurements involving glass take on two unique technical aspects. Some applications require looking at the glass surface. This can be complex since at shorter wavelengths glass is transparent to $I R$ and at longer wavelengths glass is reflective. Other applications involve

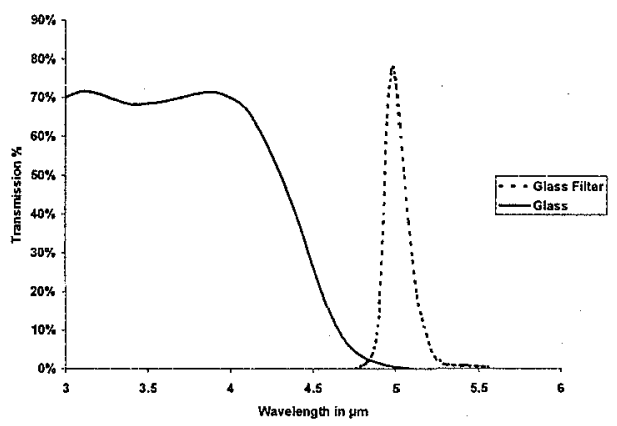

Fig. 6. Spectral characteristics of glass IR transmission below $4.5 \mu \mathrm{m}$ and opacity at $5 \mu \mathrm{m}$ looking through the glass at objects encased in glass. In this case, you want to eliminate the semi-transparent nature of the glass at certain wavelengths.

\section{Measuring glass surfaces}

Measuring the surface temperature of glass is a common practice in the light bulb, bottling and windshield industries. Temperature measurement is critical to the tempering process and is thus widely used in a non-contact manner. Research has shown that by operating at a wavelength centered on $5.0 \mu \mathrm{m}$ provides the best results. At this wavelength, you are just above the region where glass is transmissive, but below the region where glass becomes highly reflective.

The thermal image shown has been taken with a glass filter that operates from 4.95$5.05 \mu \mathrm{m}$. Having this narrow spectral characteristic provides highly accurate measurements at higher temperatures. A wider band pass filter can provide lower temperature measurements, down to near ambient temperatures.

\section{Measuring through glass}

The ability to measure through glass can be useful when looking at objects which are encased in glass such as the filaments inside a light bulb or heater element. Glass transmits IR fairly well in the shortwave region up to about $4.5 \mu \mathrm{m}$. The ideal filter for this application would be a $4.5 \mu \mathrm{m}$ "cut-off" filter. For applications involving higher temperatures, a flame suppression filter can be used. Being centered at $3.9 \mu \mathrm{m}$ the filter looks at a region where glass is quite transmissive. For lower temperature applications, the plastics filter can be used, as this filter also operates in a region where glass is highly transmissive.

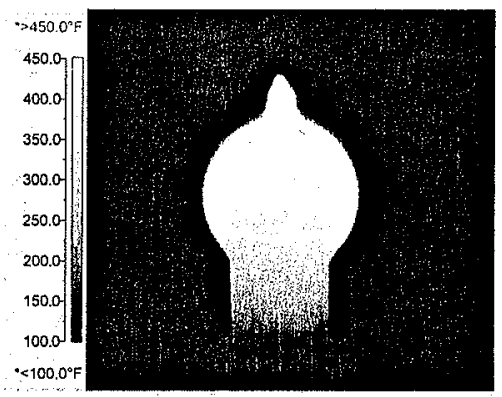

Fig. 7. Image of light bulb with glass filter eliminates "seeing through" the glass

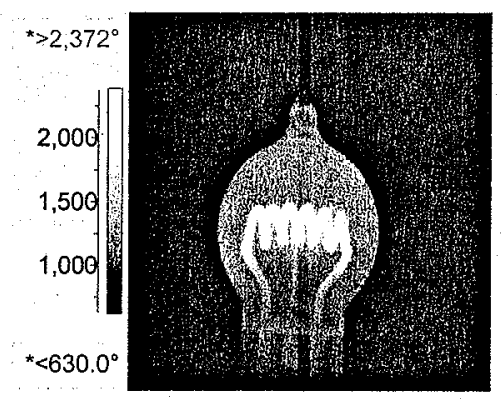

Fig. 8. Image of light bulb filament looking through the glass with $3.9 \mu \mathrm{m}$ filter 


\section{Imaging transparent gasses}

Most gasses appear transparent to an IR system. The ability to be able to "see" gasses with an IR system can be very useful in a wide range of applications involving leak detection. In a leak detection application, you need to choose a gas that has absolution bands in the appropriate wavelengths in the IR spectrum, is inexpensive and not harmful to the environment. $\mathrm{CO}_{2}$ is an ideal gas meeting these criteria. $\mathrm{CO}_{2}$ has an absorption band at $4.28 \mu \mathrm{m}$, which can be viewed with a typical shortwave camera. FPA cameras are ideal for using this type of filter since they have a larger detector integration time, providing the needed sensitivity for this application. In order to see the gas, you must have a warm scene behind the gas for the gas to absorb energy from. When the gas absorbs the energy from the warm background, it blocks the energy going to the camera. This appears as cooler regions in the image, thus making the gas appear "black" on a black and white image.

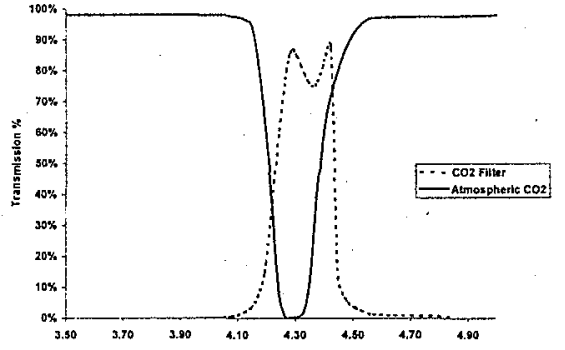

Fig.9. Spectral transmission of $\mathrm{CO}_{2}$ gas shows opaque region at $4.28 \mu \mathrm{m}$

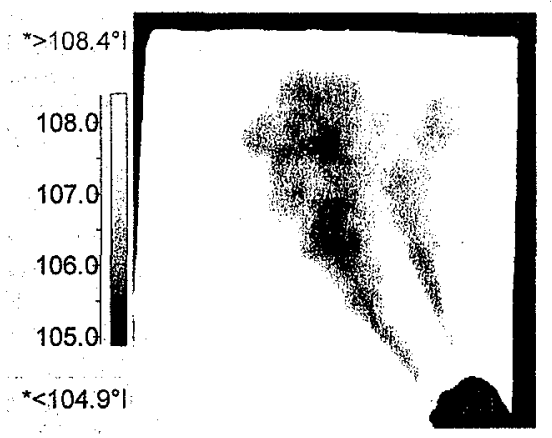

Fig. 10. Thermal image of $\mathrm{CO}_{2}$ gas leak from an automotive heater core

\section{Special considerations when using filters}

The use of filters within calibrated thermal imaging systems comes with some drawbacks. In almost all cases, the use of a filter limits the total energy going to the IR detector. When working with high temperatures, this is not considered to be a problem. However, when working with lower temperatures, this causes the IR signal-to-noise ratio to go down, resulting in poorer sensitivity and accuracy. A properly designed IR camera can help to mitigate these factors by adding features for placing the filter at the correct point in the optical path and having temperature sensors to monitor the filter's temperature. It is important to understand the limitations that a filter places on the performance of an IR system prior to putting one into use.

\section{Conclusion}

Many applications problems, which we see in infrared, can be solved by utilizing spectral filters. With an understanding the spectral characteristics of the various objects we view, we can enhance the capabilities of an IR camera by "tuning" it's performance with filters. The use of filters opens new avenues for viewing plastics, glass, process furnace components and leaking gasses. 